\title{
Über Zeitlichkeit und Ewigkeit
}

\author{
Reflexionen im Gespräch mit Augustin
}

Luzius Müller

\section{Alterung, Endlichkeit und Sterblichkeit: akzeptieren oder überwinden?}

Alles Leben ist endlich. Bezüglich individueller organischer Lebensvollzüge äussert sich die Endlichkeit im Prozess der Alterung und im schliesslichen Absterben des je individuellen Lebens. Der Tod ist der manifeste Ausdruck der Endlichkeit allen Lebens.

Schon seit etlichen Jahren fördern Untersuchungen zur pharmakologischen, biochemischen und gentechnologischen Beeinflussung des Alterungsprozesses grundlegende Erkenntnisse zu Tage. So gelang beispielsweise bei Caenorhabditis elegans, einem Fadenwurm und beliebten biologischen Modellorganismus, eine Verlängerung der Lebensspanne mittels kalorienarmer Ernährung. ${ }^{1}$ Arbeiten, welche solche Verzögerungen des Alterungsprozesses und Verlängerungen der Lebensspanne beschreiben, berechtigen jedoch nicht zu Träumereien über Unsterblichkeit (ob diese Träumereien in den Hoffnungen der Promotoren der anti-aging-medicine oder aber in den Ängsten ihrer Kritiker in Form von Albträumen wirkmächtiger sind, sei dahingestellt). Erstens sind Menschen keine Fadenwürmer. Zweitens bleibt individuelles Leben sterblich, auch wenn sein Alterungsprozess beeinflusst werden kann. Drittens ergibt eine Beeinflussung des Alterungsprozesses bloss eine Verschiebung oder Verzögerung desselben, aber nicht seine Aufhebung.

Der Traum vom Ende der Endlichkeit und von einer Therapie gegen den Alterungsprozess ist alt und findet beispielsweise im schon in der Antike bekannten Motiv des Jungbrunnens seinen Niederschlag. Die moralische Beurteilung dieses Wunschtraumes fiel und fällt unterschiedlich aus, denn die Tatsache der Endlichkeit allen

\footnotetext{
1 Vgl. Nicholas A. Bishop et al., Two neurons mediate diet-restriction induced longvity in C. elegans, in: Nature 447, 2007, 545-549; Siler H. Panowski et al., PHA-4/ Foxa mediates diet-restriction-induce longvity of C. elegans, in: Nature 447 (2007), $550-555$.
} 
Lebens an sich kann zu unterschiedlichen ethischen Ableitungen und unterschiedlichen Formen des Umgangs mit derselben führen.

So sind beispielsweise die moralischen Stossrichtungen der die Endlichkeit thematisierenden Sentenzen denkbar vielfältig. Die gängige Interpretation des memento mori der clunyazensischen Klosterfrömmigkeit beispielsweise will dem Menschen seine Endlichkeit und Sterblichkeit vor Augen führen und bringt die menschliche Existenz somit ausschliesslich in Hinblick auf ihre Nicht-Existenz zur Sprache. Diese Interpretation kann zu einer Ab- oder Aufwertung der endlichen Lebenszeit führen. Die endliche Lebenszeit kann angesichts des Todes zur quantité négligeable schrumpfen, was zu einer passiv-quietistischen Lebenshaltung führen mag. Die Begrenztheit des Lebens kann aber umgekehrt seinen Wert und seine Bedeutung als `knappes Gut steigern und zu sittlichem Aktivismus auffordern.

Güterethischen Ansätze hinsichtlich eines guten Lebens, Alterns und Sterbens, welche die aristotelische Tugend der Mässigung propagieren, müssen daher mit starken ethischen Zusatzannahmen operieren. ${ }^{2}$ Solche Konzepte zur Lebensgestaltung mögen als Klugheitsregeln praktische Evidenz besitzen. Sie haben aber in ihrer moralischen Partikularität keinerlei kategorischen Anspruch und stellen also keine allgemeingültigen Argumente gegen eine antiaging-medicine oder für eine Begrenzung von Medizin im Alter zur Verfügung. ${ }^{3}$

\section{Für immer, aber in welchem Zustand?}

In eine ganz andere Richtung weist die Frage, wie bzw. in welchem Zustand Menschen denn eigentlich sehr viel älter werden wollten vorausgesetzt, die medizinischen und genetischen Technologien zur Sistierung des Alterungsprozesses wären tatsächlich vorhanden. Der Mensch ist vom ersten Moment seiner Existenz an der Zeitlichkeit, Entwicklung, Veränderung und Alterung unterworfen. Es stellt sich daher die Frage, welcher Zustand dieser Entwicklung denn nun 'konserviert» werden sollte, vorausgesetzt es liesse sich ein beliebiger physischer Entwicklungszustand konservieren. Gewiss findet im Motiv des Jungbrunnens der Wunsch nach physischer Jugendlichkeit seinen Ausdruck, aber geht damit auch der Wunsch nach jugendli-

2 Beispielsweise: Daniel Callahan, Setting Limits. Medical Goals in an Aging Society, New York 1987.

3 Luzius Müller, Grenzen der Medizin im Alter? Individualethische und sozialethische Diskussion einer Begrenzung von Medizin im Alter, Zürich 2010, 280-284. 
chem Ungestüm einher? Ist aber die Überwindung jugendlichen Ungestüms nur eine Frage der geistigen Erfahrung, welche sich bei Sistierung der physischen Alterung auch mittels eines jugendlichen Leibes sammeln liesse, oder ist sie eine Frage der biochemischen Entwicklung des Gehirns bzw. des hormonellen Haushalts und also auch eine Frage physischen Entwicklung?

Der mit solchen Überlegungen aufgeworfenen Frage kann im Medium einer ganz anderen Thematik nachgegangen werden: Die Vorstellungen von Unsterblichkeit und ewigem Leben haben traditioneller Weise ihren Ort in der christlich-religiösen Rede von der Auferstehung der Toten in ein unvergängliches Wesen (z.B. 1. Kor 15, 35-58). In welches Alter, in welchen Zustand der menschlichen Entwicklung hinein soll nun der Tote auferstehen? Die Tradition kennt verschiedene Antworten auf diese Frage. In Augustinus De civitate Dei wird von Christus als vom "vollkommenen Manne" gesprochen, in dessen Alter und leiblichen Zustand hinein die Toten auferstehen würden. ${ }^{4}$ Augustinus beschreibt das Alter von 30 Jahren (das vermutete Sterbe- und also auch Auferstehungsalter Jesu) als das Alter jugendlicherVollreife. ${ }^{5}$ Es besteht aber auch dieVorstellung, die Toten würden so auferstehen, wie sie je gestorben sind. ${ }^{6}$ Eine hochaltrige Gesellschaft wird sich eher an Augustinus' Ansatz orientieren wollen ...

Die Frage nach dem Zustand und Alter der Auferstandenen rührt an ein systematisch-theologisches Grundproblem: Sofern eine theologische Anthropologie den Menschen sub conditione aeternitatis versteht, seine Endlichkeit und Sterblichkeit also aufgehoben weiss in der göttlichen Ewigkeit, stellt sich die Frage, welches Recht der menschlichen Zeitlichkeit, Entwicklung,Veränderung und Alterung sub conditione aeternitatis zukommen kann. Diese Problemlage mag dazu beigetragen haben, dass die christliche Überlieferung zwar lebenspraktische Regeln, aber kein ausgewiesenes dogmatisches Konzept zum Topos der Alterung kennt. Unter den Bedingungen einer eschatologischen Vollendung des Menschen in der Auferstehung muss die Wahrnehmung der menschlichen Alterung zwangsläufig entfallen. Eine eschatologisch angelegte Anthropologie, die das Lebensganze des Menschen sub conditione aeternitatis betrachtet, erkennt diesen zwangsläufig, als alterslos, ahistorisch, als ewiges und vollkommenes Geschöpf Gottes - und also nicht als eine zeitliche, alternde

\footnotetext{
4 Augustinus, De civitate Dei, zweiundzwanzigstes Buch, Kapitel 18.

5 Ebd., Kapitel 15.

${ }_{6}$ Beispielsweise: Ernst Joseph Gustav De Valenti, Eschatologie oder die Lehre von den letzten Dingen, Bern/Basel 1840, 65.
} 
und sich verändernde (insbesondere auch leibliche) Existenz. Wohl ist theologischerseits die Zeitlichkeit und Endlichkeit des Menschen im Blick, aber eben als existenzielle Grundkategorie, unabhängig von seiner materialen Entwicklung,Veränderung und Alterung.

\section{Ewigkeit als Ende der Zeitlichkeit in den Confessiones Augustinus'}

Eine zum Klassiker gewordene Auseinandersetzung mit der Frage nach der Bedeutung der menschlichen Zeitlichkeit im Gegenüber zur göttlichen Ewigkeit führt der Kirchenvater Augustinus in seinen Bekenntnissen. ${ }^{7}$ Als Autobiographie verfasst, spielen hier Zeitlichkeit, Veränderung, Entwicklung und Alterung eine wesentliche Rolle. Zugleich sind die Confessiones als eine betende Zwiesprache des Autors mit dem ewigen Gott konzipiert, sodass Zeitlichkeit und Ewigkeit miteinander konfrontiert werden. Diese Konfrontation artikuliert sich schon zu Beginn des ersten Buches:

"Gross bist Du, Herr, und hoch zu preisen [...]. Und preisen will Dich der Mensch [...], der herumschleppt sein Sterbewesen. [...] [D]enn geschaffen hast Du uns zu Dir, und ruhelos ist unser Herz, bis dass es seine Ruhe hat in Dir. ${ }^{8}$

Eine prägnante Formulierung dieser Konfrontation von Zeitlichkeit und Ewigkeit in Gottes Schöpfungswerk findet sich noch in den einleitenden Worten:

"Was also bist $\mathrm{Du}$, mein Gott? [...] Du, über alles bist $\mathrm{Du}$ der Hohe, der Gute, der Mächtige, der Allmächtige [...], der Unwandelbare, der alles wandelt: nie bist $\mathrm{Du}$ neu, nie bist $\mathrm{Du}$ alt und erneuerst doch alles. «"

Das Leitthema des Werkes ist die Suche nach der Ruhe in Gott. ${ }^{10}$ Es macht klar, dass der Zeitlichkeit des Menschen angesichts der Ewigkeit Gottes eine untergeordnete Bedeutung zukommen muss: In der Gottesruhe kommt alle Veränderung und Entwicklung zu ihrem Ende. Die in den Büchern 1-9 dargestellte Biographie wird als

\footnotetext{
7 Im vorliegenden Essay findet die Übersetzung Joseph Bernharts Verwendung, deren stilistische Qualität den Autoren überzeugt: Joseph Bernhart, Augustinus Bekenntnisse. Zweisprachige Ausgabe, Frankfurt a.M. 1987.

8 Liber primus $1,1$.

9 Ebd., 4,4.

${ }^{10}$ Ebd., 5,5.
} 
ein unruhiges Irren in der Zeit verstanden, auf der ständigen Suche nach der Ruhe in Gottes Ewigkeit und Unveränderlichkeit.

Mit dem elften Buch setzt eine Auslegung der biblischen Schöpfungsgeschichte ein, welche in die bekannte Erörterung der Zeit mündet. Die Thematik der Zeit wird eingeführt anhand der Frage, was Gott vor der Schöpfung getan habe. Diese Frage weist Augustinus mit der Annahme zurück, dass der ewige Gott mit der Schöpfung die Zeit erschaffen habe, dass also in Gottes Ewigkeit kein Vorher und Nachher, keine Zeit sei. Ewigkeit wird zeitlos gedacht.

„Denn woher denn der Fluss dieser unzähligen Jahrhunderte, die nicht Du geschaffen hättest, der Du doch der Urheber und Gründer aller Jahrhunderte bist? Oder was für Zeiten wären da gewesen, die nicht Du gegründet hättest? «"11

Die Erörterung der Zeit leitet über zur Erkenntnis, dass sie ihre Wirklichkeit nur im Bewusstsein des Menschen hätte und sich aus Erinnerung und Erwartung ergebe, dass die Gegenwart daher der ausdehnungslose Moment des Umschlags von Zukunft in Vergangenheit sei. Im Kontext der Confessiones dient diese an sich beachtenswerte Theorie der Zeit dazu, die Bedeutung der Zeit und Zeitlichkeit im Gegenüber der Ewigkeit Gottes herabzumindern ("Zerfahrenheit ist mein Leben ${ }^{12}$ ) und also das Leitmotiv der Sehnsucht nach der Ruhe in der Ewigkeit Gottes erneut aufzugreifen.

"Noch aber schwinden meine Jahre in Seufzen dahin, und Du nur bist mein Trost, Herr, mein Vater, und Du bist ewig; ich aber splittere in Zeit und Zeit [sic] und kenne ihre Ordnung nicht, und im aufgeregten Umbestand der Dinge werden meine Gedanken, wird das tiefste Leben meiner Seele hierhin, dorthin gezerrt, bis ich, in der Glut Deiner Liebe zu lauterem Fluss geschmolzen, in Dir ein ungeteiltes Eines werde. ${ }^{13}$

Die Confessiones können der Zeitlichkeit angesichts der Ewigkeit keine Bedeutung zukommen lassen. Das Zeitliche findet seine Berechtigung nur in der Suche nach der Ewigkeit Gottes. Die zeitlichzerdehnte und zersplitterte Existenz wird schliesslich geschmolzen und aufgelöst in der ungeteilten, ewigen Einheit Gottes.

Aber die Confessiones sind dennoch als Autobiographie konzipiert. Die permanent betonte Bedeutungslosigkeit der Zeitlichkeit steht in einem gewissen Widerspruch zur ausführlichen Darlegung des

\footnotetext{
${ }^{11}$ Liber undecimus 13,15.

${ }^{12}$ Ebd., 29,39.

${ }^{13}$ Ebd.
} 
eigenen Lebensweges mit all seinen Entwicklungen und Veränderungen. So sind sie eine eindrückliche Darstellung der Schwierigkeit, Zeitlichkeit, Veränderung, Entwicklung und Alterung des Menschen angesichts der Ewigkeit Gottes zu denken. Zeitlichkeit und Endlichkeit - Konstitutiva der menschlichen Existenz - können sub conditione aeternitatis nicht existieren.

"Wer es versteht, bekenne Dir, und wer es nicht versteht, bekenne Dir! «14

- Luzius Müller ist Chemiker und evangelischer Theologe, er arbeitet als Universitätspfarrer an der Universität Basel. Zu seinen Arbeitsgebieten gehört insbesondere die theologische Ethik.

${ }^{14}$ Ebd., 31,41. 